\title{
Electron correlations, spontaneous magnetization and momentum density in quantum dots
}

\author{
A. Bansil, D. Nissenbaum, B. Barbiellini \\ Physics Department, Northeastern University, Boston MA 02115 \\ R. Saniz * \\ Departamento de Ciencias Exactas, Universidad Católica Boliviana, Casilla \#5381, Cochabamba, Bolivia
}

\begin{abstract}
The magnetization of quantum dots is discussed in terms of a relatively simple but exactly solvable model Hamiltonian. The model predicts oscillations in spin polarization as a function of dot radius for a fixed electron density. These oscillations in magnetization are shown to yield distinct signature in the momentum density of the electron gas, suggesting the usefulness of momentum resolved spectroscopies for investigating the magnetization of dot systems. We also present variational quantum Monte Carlo calculations on a square dot containing 12 electrons in order to gain insight into correlation effects on the interactions between like and unlike spins in a quantum dot.
\end{abstract}

PACS numbers: 73.22.Dj, 75.75+a, 75.10-b

\section{INTRODUCTION}

As the need for nano-structures for technological applications grows, the ability to probe and understand the electronic properties of these systems becomes of paramount importance [1, 2, 3]. In this connection, quantum dots (QDs), which can be viewed as artificial "atoms", offer unique opportunities as a nanoscale laboratory for investigating the behavior of small numbers of electrons and how the interplay between correlation and confinement effects in such systems can give rise to novel phenomenon such as spontaneous spin polarization of the electron gas [4, 5, 6, 7]. These and related questions have been the subject of considerable interest in the recent literature [8, 9].

Here we discuss how the electronic structure of QDs can be modeled theoretically for the purpose of gaining a handle on the essential phenomenology of their magnetic properties. The exactly solvable model Hamiltonian introduced in Ref. [4] is considered first. The model of Ref. [4] assumes a single effective interaction parameter, $U$, which gives the energy penalty for creating a pair of electrons with opposite spins. Despite its simplicity, this model produces considerable richness in its behavior and, in particular, it predicts oscillations in spin polarization with QD radius at a fixed electron density. We delineate the signature of spin polarization in the electron momentum density (EMD), thus setting the stage for the application of momentum resolved spectroscopies as a window for investigating the magnetic properties of QDs.

Although exactly solvable many-body models are of an intrinsic interest, it is important to understand the

*Present address: Department of Physics and Astronomy, Northwestern University, Evanston, Illinois 60208 nature of the parameters involved in such models via accurate first principles computations. In this connection, we have carried out variational quantum Monte Carlo (VQMC) calculations in the interacting electron gas. Results for a 12 electron system confined within a square QD are presented. The computed pair correlation functions for like and unlike spins are used to deduce the effective value of the parameter $U$, which enters the model Hamiltonian of Ref. [4].

An outline of this article is as follows. The introductory remarks are followed in Section II by an overview of the model Hamiltonian formalism of Ref. [4]. Section III presents the VQMC approach and considers the example of a 2D square QD. Section IV presents a few concluding remarks.

\section{A MODEL HAMILTONIAN FOR QUANTUM DOTS}

Insight into properties of QDs can be obtained by considering the relatively simple model Hamiltonian [4]

$$
\begin{aligned}
\hat{H} & =\hat{H}_{0}+\hat{H}_{1} \\
& =\sum_{\nu \sigma} \epsilon_{\nu}^{0} a_{\nu \sigma}^{\dagger} a_{\nu \sigma}+\frac{1}{2} U \sum_{\nu \nu^{\prime} \sigma} a_{\nu \sigma}^{\dagger} a_{\nu^{\prime}-\sigma}^{\dagger} a_{\nu^{\prime}-\sigma} a_{\nu \sigma}
\end{aligned}
$$

where $a_{\nu \sigma}^{\dagger}$ and $a_{\nu \sigma}$, respectively, are the creation and annihilation operators for the one-particle state $\phi_{\nu \sigma}$ with eigenvalue $\epsilon_{\nu}^{0}$. The first term $\left(\hat{H}_{0}\right)$ describes the noninteracting system. The interaction in the second term $\left(\hat{H}_{1}\right)$ is restricted to electrons of opposite spins. The parameter $U$ here can be viewed as an average energy penalty for two electrons to possess opposite spins in the QD. It is of course energetically advantageous for electrons to possess the same spin because that allows the Coulomb energy to be lowered as the electrons are kept apart by the Pauli exclusion principle. 


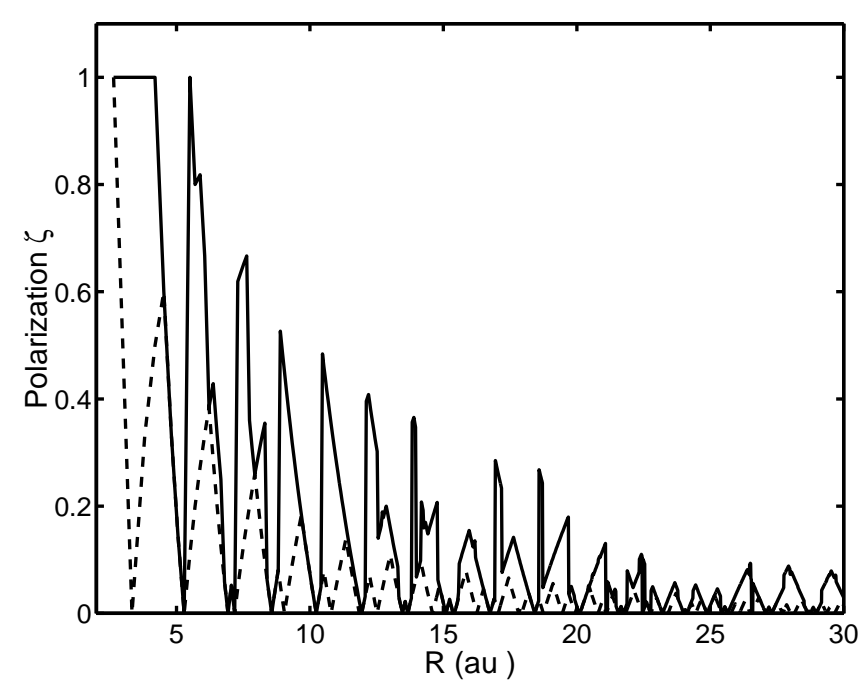

FIG. 1: Spin polarization per particle, $\zeta=\left(N_{\uparrow}-N_{\downarrow}\right) / N$, as a function of the dot radius $R$. Solid line: interacting case; dashed line: weakly interacting case, $U \rightarrow 0$. Simulations are based on the model Hamiltonian of Eq. 11 using parameters discussed in the text.

The model Hamiltonian of form (11) can be solved exactly. The solution for the many-body wavefunction has the form of an unrestricted Hartree-Fock wavefunction

$$
\Psi=D^{\uparrow} D^{\downarrow},
$$

where up and down arrows denote spin indices and $D^{\sigma}=$ $\left|\phi_{i, \sigma}\left(\mathbf{r}_{j}\right)\right|$ is the Slater determinant formed by one particle states $\phi_{i, \sigma}$. Note that this solution is a spin eigenfunction and does not suffer from the so-called spin contamination problem [4]. The total energy is given by

$$
E=\sum_{\nu \sigma}\left(\epsilon_{\nu}^{0}+\frac{1}{2} U N_{\sigma}\right) f_{\nu \sigma}
$$

where $f_{\nu \sigma}$ denotes the Fermi occupation function. For a given total number of particles $N$, minimization of Eq. 3 yields a set of nonlinear equations for the populations, $N_{\uparrow}$ and $N_{\downarrow}$, of the up and down spins, respectively. The resulting splitting in energy for states of opposite spin is

$$
\Delta=U\left(N_{\uparrow}-N_{\downarrow}\right),
$$

and it is uniform, i.e., it does not depend on the quantum number $\nu$. The average polarization per electron is

$$
\zeta=\left(N_{\uparrow}-N_{\downarrow}\right) / N .
$$

The degeneracy between up and down spin electrons is thus lifted and the shell filling depends on the value of $\Delta$, which is determined by the interaction strenght $U$.

Fig. 1 presents a few illustrative results based on the model Hamiltonian of Eq. 1 The details of the specific parameters used are as follows. The non-interacting
Hamiltonian is taken to be a $3 \mathrm{D}$ spherical well with potential, $V(r)=-8.62 \mathrm{eV}$, for $r \leq R$, and $V(r)=0$, otherwise. The electron density $n$ in the QD is kept fixed corresponding to $r_{s}=5$, where $r_{s}$ is the standard parameter given by, $n\left(4 \pi r_{s}^{3} / 3\right)=1$. The QD radius $R$ and the number $N$ of electrons are thus related via $N=\left(R / r_{s}\right)^{3}$. $r_{s}=5$ gives a relatively low density, enabling consideration of a wide range of QD radii. The choice of $U$ is more tricky since correlations in QDs are not well understood. However, on the basis of arguments involving a screened Coulomb interaction, Ref. [4] estimates $U=27.13 \mathrm{meV}$ when $N=96$ or $R=12.11 \AA$ [10].

Fig. 11 shows the average polarization $\zeta$ as a function of the QD radius $R$ for $r_{s}=5$. As $R$ increases and electrons are added, spin polarization $\zeta$ reaches a peak each time a shell is half filled with up-spin electrons and falls to zero when the shell is completed with down-spin electrons, yielding a sequence of "magic numbers", i.e., $N$ values for which the QD magnetization vanishes. The oscillations in spin polarization are damped with increasing QD size and in the high $R$ limit a paramagnetic homogeneous electron gas is recovered. Fig. 1 also shows that the interaction parameter $U$ can give large deviations in $\zeta$ from a simple Hund's rule filling. This is because the magnetic energy splitting changes with each added electron in order to minimize the total energy given by Eq. 3 .

In connection with spontaneous magnetization, it is useful to consider the Stoner model, which is usually invoked for predicting ferromagnetism in metals [1], although it has also been applied more recently to discuss magnetism of nanosystems [6]. In the Stoner model, ferromagnetism results if

$$
I D(\mu) \geq 1,
$$

where $D(\mu)$ is the density of states (DOS) per unit cell of the spin compensated system at the Fermi level $\mu$ and $I$ is the Stoner parameter, which gives the gain in potential energy associated with the occurrence of the ferromagnetic state. Within the Density Functional Theory (DFT), I can be computed using the wavefunctions of the system at $\mu$ [11]. In the case of the homogeneous electron gas $I$ reduces to $[6$

$$
I=\frac{8\left[\epsilon_{x c}^{F}\left(r_{s}\right)-\epsilon_{x c}^{P}\left(r_{s}\right)\right]}{9\left(2^{4 / 3}-2\right)}
$$

where $\epsilon_{x c}^{F}$ and $\epsilon_{x c}^{P}$ are the exchange-correlation energy per electron in the ferromagnetic and the paramagnetic electron gas, respectively. Interestingly, for the model Hamiltonian of Eq. 10 the connection between the average energy penalty $U$ for having a pair of electrons with opposite spins and the Stoner parameter $I$ is given as 12 .

$$
I=U N \text {. }
$$

Eq. 6] makes it clear that, for a finite $I$, singularities in the DOS can be expected to induce spontaneous magnetization [6]. In QDs with high symmetry (e.g., spherical 


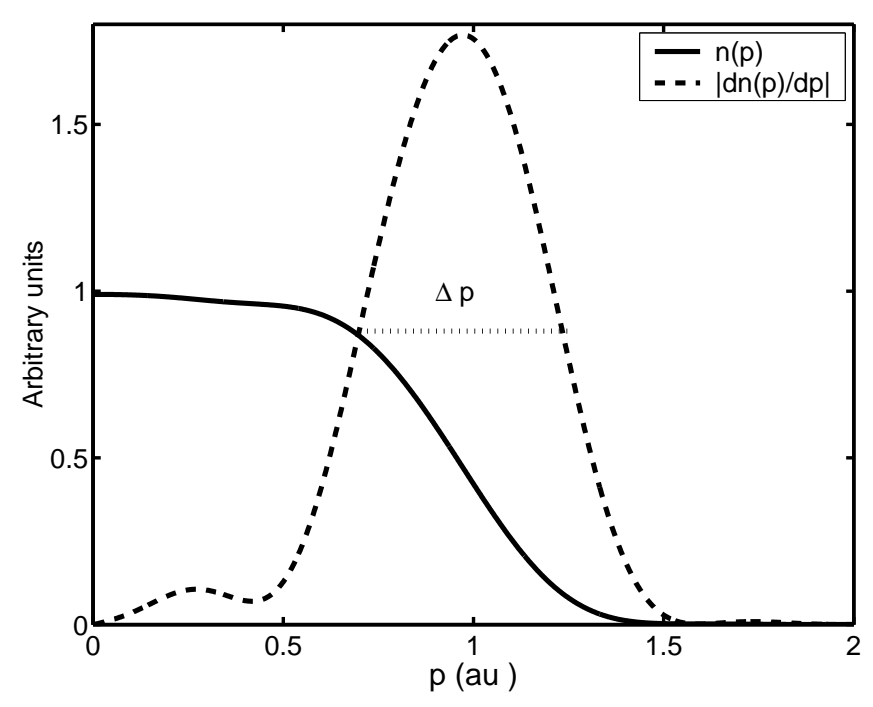

FIG. 2: Typical EMD in a QD, $n(p)$, and the magnitude of its first derivative, $\left|n^{\prime}(p)\right|$. The position of the peak in $\left|n^{\prime}(p)\right|$ defines the QD "Fermi momentum", while its full-width-athalf-maximum defines $\Delta p$.

or cubic dots), symmetry related degeneracies will generally enhance DOS peaks. On the other hand, symmetry breaking effects [5] and disorder [13] will smear out DOS peaks and reduce the tendency for the system to magnetize spontaneously.

We discuss next the EMD with an eye towards identifying signatures of spin polarization in a QD. The EMD is defined by

$$
n(\mathbf{p})=(2 \pi)^{-1} \int_{-\infty}^{\infty} d \omega f(\omega) A(\mathbf{p}, \omega)
$$

where $f$ is the Fermi function and

$$
A(\mathbf{p}, \omega)=-2 \operatorname{Im} G^{R}(\mathbf{p}, \omega),
$$

is the spectral function. The one particle Green's function $G^{R}(p, \omega)$ and its imaginary part can be evaluated exactly for the model Hamiltonian of Eq. 11 The typical behavior of the EMD and its derivative is shown in Fig. 2 The region of rapid variation in $n(p)$ can be characterized via the position, $p_{\mathrm{F}}$, of the peak in $\left|n^{\prime}(p)\right|$ and the associated full-width-at-half-maximum, $\Delta p$. In the bulk limit in a metallic system, the EMD in general contains Fermi surface (FS) breaks in the first Brillouin zone (BZ) and at the Umklapp images of the FS in higher BZs. Correspondingly, the first derivative of the EMD develops $\delta$-function peaks. Although in a finite system there cannot be breaks in the EMD, we may nevertheless refer to $p_{\mathrm{F}}$ loosely as the QD "Fermi momentum" for simplicity.

Fig. 3 shows the simulated behavior of $\Delta p$ in a QD as a function of the radius $R$ at a fixed electron density. $\Delta p$ is seen to display peaks, which are well correlated with

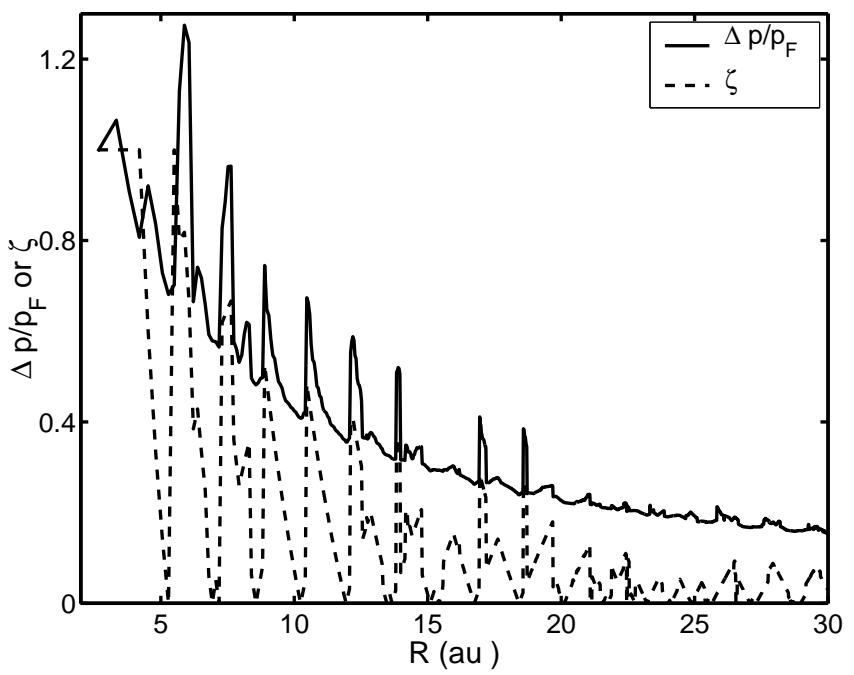

FIG. 3: $\Delta p$ in reduced units of $p_{\mathrm{F}}$ (solid line), and spin polarization $\zeta$ (dashed) vs. QD radius $R$. Simulations are based on the model Hamiltonian of Eq. 11 using parameters discussed in the text.

those in the magnetization $\zeta$. The reason for this correlation between $\Delta p$ and $\zeta$ is that in the polarized system, in effect, there are two separate momentum distributions for the up and down spin electrons. The two associated "Fermi" momenta then give rise to two different peaks in $\left|n^{\prime}(p)\right|$, which appear as increased broadening of $\Delta p$ in the total momentum density of the interacting system. In fact the $R$ dependence of $\Delta p$ can be fitted as [4]

$$
\Delta p / p_{\mathrm{F}}=\Delta p_{0} / p_{\mathrm{F}}+c \Delta\left(\zeta-\zeta_{0}\right) R
$$

where $\zeta_{0}$ is the spin polarization in the weakly interacting case $(U \rightarrow 0)$, and $c$ is a constant, which depends on various QD parameters. Eq. 11] can be used to extract the polarization $\zeta$ from the measured R-dependence of $\Delta p$. These considerations indicate that peaks in $\Delta p$ provide a distinct signature of polarization of a $\mathrm{QD}$ and that spectroscopies sensitive to momentum density can play a useful role in this connection [14]. These results argue for investigations of the QDs using Compton scattering and positron annihilation experiments.

It should perhaps be noted that Compton scattering and positron annihilation spectroscopies have developed by now into standard probes of the EMD in materials. Recent positron annihilation measurements on CdSe QDs show that the effect of quantum confinement results in an increased width $\Delta p$ of the Fermi edge in momentum space. The width $\Delta p$ seems to follow an inverse square law $1 / d^{2}$ with particle diameter $d$ 15, 16], in contrast to the $1 / d$ law expected for the confined homogeneous electron gas [17. 


\section{A QUANTUM MONTE CARLO CALCULATION}

The discussion of the preceding section is based on the Hamiltonian of Eq. 11 and it is thus limited by the form of the Hartree-Fock many-body wavefunction given by Eq. 2 We now consider a more general many-body wavefunction and apply the VQMC approach [18] to focus particularly on understanding the nature of the effective interaction between electrons of the same and opposite spins in a QD. The specific wavefunction used is

$$
\Psi=J D^{\uparrow} D^{\downarrow}
$$

where

$$
J=\exp \left(\sum_{i<j} u_{i j}\right)
$$

is the Jastrow factor, which is expressed in terms of a product involving two-body correlation factors, $u_{i j}$. The $u_{i j}$ must fulfill the so-called cusp condition, i.e. the singularities associated with the kinetic energy must cancel those arising from the Coulomb potential in the microscopic Hamiltonian. A simple form is given by 18

$$
u(r)=\frac{r}{1+\beta r}
$$

where $\beta$ is a variational parameter. When $J=1$ or $\beta \rightarrow \infty, \Psi$ reduces to the form of an unrestricted HartreeFock wave function.

The average repulsion energy between two electrons can be expressed as an integral of the spin resolved radial pair correlation function

$$
g_{\sigma, \sigma^{\prime}}(r)=\frac{1}{n N_{\sigma}} \sum_{i \neq j} \delta_{\sigma, \sigma_{i}} \delta_{\sigma^{\prime}, \sigma_{j}} \int \delta\left(r-r_{i j}\right)|\Psi(\xi)|^{2} d \mathbf{R}
$$

where $\mathbf{R}=\left(\mathbf{r}_{1}, \ldots, \mathbf{r}_{N}\right), \xi=\left(\mathbf{R}, \sigma_{1}, \ldots, \sigma_{N}\right), r_{i j}=\left|\mathbf{r}_{i}-\mathbf{r}_{j}\right|$, $\delta_{\sigma, \sigma^{\prime}}$ is the spin projector, and $n$ is the average electron density. The pair interaction energy between two electrons of like spins is then given by

$$
V_{\sigma, \sigma}=\frac{1}{\left(N_{\sigma}-1\right)} \int \frac{n g_{\sigma, \sigma}(r)}{r} d \mathbf{r}
$$

and for electrons of opposite spins is

$$
V_{\sigma,-\sigma}=\frac{1}{N_{\sigma}} \int \frac{n g_{\sigma,-\sigma}(r)}{r} d \mathbf{r} .
$$

The energy penalty $U$ for having a pair of electrons with opposite spins can therefore be obtained from the average

$$
U=\frac{1}{2} \sum_{\sigma}\left(V_{\sigma,-\sigma}-V_{\sigma, \sigma}\right) .
$$

As an example, we have investigated a QD consisting of 12 electrons enclosed in a $2 \mathrm{D}$ square well of size $l=\pi$

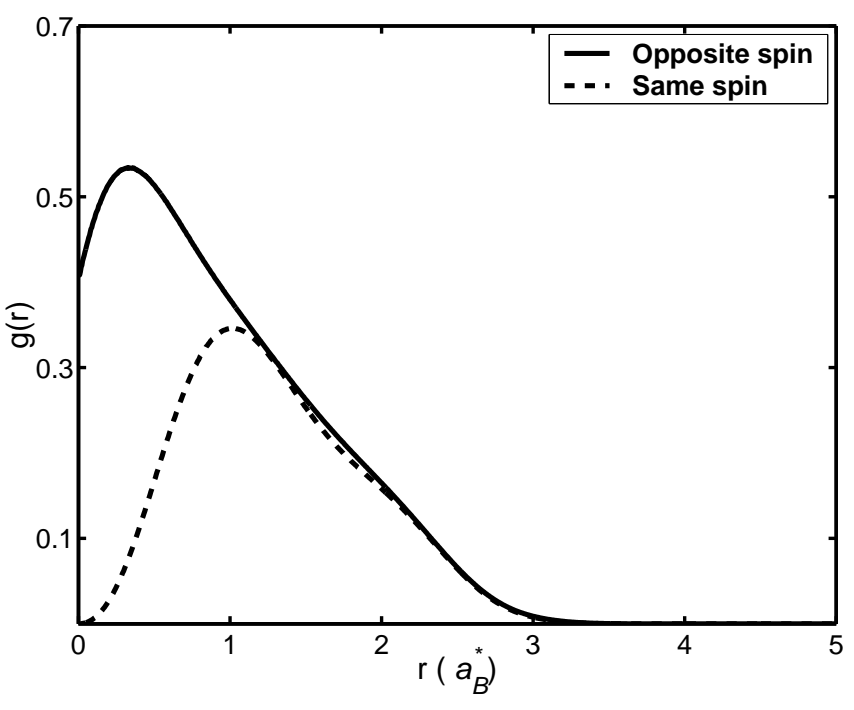

FIG. 4: Radial pair correlation functions $g_{\sigma, \sigma}(r)$ (dashed line) and $g_{\sigma,-\sigma}(r)$ (solid line) for 12 electrons enclosed in a 2D square well of size $l=\pi a_{B}^{*}$.

$a_{B}^{*}$ [19]. Here and elsewhere in this section, it is convenient to use the modified atomic units $a_{B}^{*}$ for length and $\mathrm{H}^{*}$ for energy, which are renormalized atomic units obtained from the effective electron band mass $m^{*}$ and the dielectric constant of the material $\varepsilon$ [20].

We describe the confinement in the $x y$ plane by an infinite hard-wall potential, therefore the single-electron states in the square QD are

$$
\phi_{n_{x}, n_{y}, \sigma}(x, y)=\frac{2}{\pi} \sin \left(n_{x} x\right) \sin \left(n_{y} y\right)
$$

For $N=12$, one obtains a closed shell system $\left(N_{\uparrow}=N_{\downarrow}\right)$ with zero net magnetization.

In the Hartree-Fock limit $(\beta \rightarrow \infty)$, a Monte Carlo calculation gives the total energy of the 12-electron QD to be $107.785 \pm 0.002 \mathrm{H}^{*}$. The parameter $\beta$ was then optimized via the Stochastic Gradient Approximation (SGA) 18]. In the SGA, at each step $n$, the value of an observable $x$ is updated with a recursive calculation of the mean:

$$
\bar{x}_{n}=\bar{x}_{n-1}-\frac{1}{n}\left(\bar{x}_{n-1}-x_{n}\right) .
$$

At the optimal $\beta=1.53$, the total energy is found to be $103.237 \pm 0.001 \mathrm{H}^{*}$ with $|d E / d \beta|<10^{-3} \mathrm{H}^{*} a_{B}^{*}$. Using this value of $\beta$, the results of a relatively noiseless calculation of the spin-dependent pair correlation functions $g_{\sigma, \sigma^{\prime}}$ are shown in Fig. 4 As expected, the distribution $g_{\sigma, \sigma}(r)$ (dashed line) is seen to vanish at $r=0$, reflecting the presence of an "exchange hole" surrounding like spins due to the Pauli exclusion principle. Electrons of unlike spins, on the other hand, still tend to avoid each other due to Coulomb repulsion, which induces a "Coulomb" or "correlation" hole in $g_{\sigma,-\sigma}(r)$ (solid line). 
Fig. [4 shows that the "hole," or the region of depleted electron density, excludes a larger number of electrons and extends to a larger distance for like spins than for unlike spins. The decrease of $g_{\sigma, \sigma^{\prime}}$ after $r \approx 1 a_{B}^{*}$ is a geometrical effect due to the finite size of the QD. The use of these correlation functions in Eqs. 16-18 yields, $V_{\uparrow \downarrow}=1.039 \mathrm{H}^{*}, V_{\uparrow \uparrow}=0.760 \mathrm{H}^{*}$, and $U=0.279 \mathrm{H}^{*}$. The corresponding values in the Hartree-Fock limit are: $V_{\uparrow \downarrow}=1.176 \mathrm{H}^{*}, V_{\uparrow \uparrow}=0.848 \mathrm{H}^{*}$, and $U_{H F}=0.329$ $\mathrm{H}^{*}$. The Jastrow wavefunction thus leads to a reduction in the energy penalty for creating a pair of opposite spins. The overall effect however is relatively small in that the effective $U$ for the Jastrow wavefunction is only $15 \%$ smaller than $U_{H F}$. These results suggest that, despite its simplicity, the model Hamiltonian of Eq. 1 is capable of providing a reasonable description of the electron gas in QDs. Moreover, we have explicitly verified the oscillations of the magnetization in the 3D-spherical quantum dots containing up to 8 electrons by performing VQMC simulations with the SGA optimization of the total energy. The oscillations in magnetization with QD radius predicted on the basis of this Hamiltonian are presumably robust to electron correlation effects missing implicitly in the Hartree-Fock form of its solution.

\section{SUMMARY AND CONCLUSIONS}

We discuss issues of spin polarization and momentum density with focus on the treatment of correlation effects in electron gas confined within a quantum dot. In this connection, we first consider selected results based on a relatively simple model Hamiltonian of Ref. [4] in which interactions are restricted via a parameter $U$ to be non-zero only for electrons of opposite spins. This model Hamiltonian is solvable exactly and admits a solution of the Hartree-Fock form. Moreover, it displays remarkable oscillations in spin polarization with dot radius, which leave distinct signature in the electron momentum density. In order to gain insight into correlation effects more generally, we have carried out VQMC calculations on a square dot containing 12 electrons using a JastrowSlater form of the many body wavefunction. The effective $U$ value for the Jastrow-Slater wavefunction is found to be only $15 \%$ smaller than for the Hartree-Fock case. On the whole, we conclude that spectroscopies sensitive to electron momentum density-Compton scattering and positron annihilation in particular-can potentially help delineate spin polarization effects in quantum dots.

This work is supported by the US Department of Energy contract DE-AC03-76SF00098, and benefited from the allocation of supercomputer time at the NERSC and the Northeastern University's Advanced Scientific Computation Center (ASCC).
[1] L. P. Kouwenhoven, C. M. Marcus, P. L. McEuen, S. Tarucha, R. M. Westervelt, and N. S. Wingreen, in Mesoscopic Electron Transport, NATO Science Series: E, Vol. 345, edited by L. L. Sohn, L. P. Kouwenhoven, G. Schön, (Kluwer, Dordrecht, 1997).

[2] S. M. Reimann and M. Manninen, Rev. Mod. Phys. 74, 1283 (2002).

[3] H. Jiang, H.U. Baranger, and W. Yang, Phys. Rev. Lett. 90, 026806 (2003).

[4] R. Saniz, B. Barbiellini, A. B. Denison, A. Bansil, Phys. Rev. B 68, 165326 (2003).

[5] I. I. Yakimenko, A. M. Bychkov, and K.-F. Berggren, Phys. Rev. B 63, 165309 (2001).

[6] N. Zabala, M. J. Puska, and R. M. Nieminen, Phys. Rev. Lett. 80, 3336 (1998) and Phys. Rev. B 59, 12652 (1999).

[7] G. L. Gutsev, S. N. Khanna, and P. Jena, Phys. Rev. B 62, 1604 (2000); for a general reference see also Quantum Phenomena in Clusters and Nanostructures, Ed. S. N. Khanna and A. W. Castleman, Jr., (Springer, New York, 2003).

[8] D. J. Reilly, T. M. Buehler, J. L. OBrien, A. R. Hamilton, A. S. Dzurak, R. G. Clark, B. E. Kane, L. N. Pfeiffer, and K. W. West Phys. Rev. Lett. 89, 246801.

[9] W. H. Li, S. Y. Wu, C. C. Yang, S. K. Lai, K. C. Lee, H. L. Huang and H. D. Yang, Phys. Rev. Lett. 89, 135504 (2002); H.Hori, T. Teranishi, Y. Nakae, Y. Seino, M. Miyake and S. Yamada, Phys. Lett. A 263, 406 (1999); H. Hori, Y. Yamamoto, T. Iwamoto, T. Miura, T. Teranishi, M. Miyake, cond-mat/0306261 to appear in Phys. Rev. B.

[10] The Coulomb matix elements and therefore $U$ in general depend on the size of the system.

[11] O. Gunnarson, J. Phys. F: Metal Phys. 6, 587 (1976).

[12] C.M. Hurd, Electrons in Metals, Krieger (Malabar, Forida, 1981).

[13] Ph. Jacquod and A. D. Stone, Phys. Rev. Lett. 84, 3938 (2000)

[14] Complex magnetic structures may be expected in dots of non-symmetric shapes, including the possibility of spiral spin arrangments and the formation of domains for dots of large sizes. It will be interesting to consider associated signatures in the momentum density. Magnetic Compton scattering with its ability to focus on specific magnetic states may prove to be a particularly useful spectroscopy in this regard (see, e.g., P. A. Montano, Yinwan Li, J. F. Mitchell, B. Barbiellini, P.E. Mijnarends, S. Kaprzyk, and A. Bansil, J. Phys. Chem. Solids, this volume).

[15] M.H. Weber, K.G. Lynn, B. Barbiellini, P.A. Sterne, A.B. Denison, Phys. Rev. B 66,R 041305 (2002).

[16] A.B. Denison, R. Meulenberg, S.W.H. Eijt, A. Van Veen, P.E. Mijnarends, B. Barbiellini, A. Bansil, C. Fischer, M.H. Weber, K.G. Lynn, Mat. Sci. Forum 445-446, 395 (2004).

[17] R. Saniz, B. Barbiellini and A. Denison, Phys. Rev. B 65, 245310 (2002).

[18] A. Harju, B. Barbiellini, S. Siljämaki, R.M. Nieminen and G. Ortiz, Phys. Rev. Lett. 79, 1173 (1997).

[19] E. Räsänen, H. Saarikoski, V.N. Stavrou, A. Harju, M.J. Puska and R.M. Nieminen, Phys. Rev. B 67, 235307 (2003).

[20] The band mass $m^{*}$ and the dielectric constant $\varepsilon$ of the 
polarizable background influence the modified atomic units: $\mathrm{H}^{*}=\left(\varepsilon^{2} / m^{*}\right) \mathrm{H}, a_{B}^{*}=\left(m^{*} / \varepsilon\right) a_{B}$, where $\mathrm{H}$ is the

Hartree energy unit and $a_{B}$ is the Bohr radius. For in- stance, in GaAs, using $m^{*}=0.067 m$ and $\varepsilon=12.4$, yields $\mathrm{H}^{*}=11.8572 \mathrm{meV}$ and $a_{B}^{*}=9.79 \mathrm{~nm}$. 